\title{
The Study and Application of the IoT in Pet Systems
}

\author{
Chung-Ming Own ${ }^{1}$, Haw-Yun Shin ${ }^{2}$, Chen-Ya Teng ${ }^{3}$ \\ ${ }^{1}$ Department of Computer and Communication Engineering, St. John's University, New Taipei City, Chinese Taipei \\ ${ }^{2}$ Department of Computer Science and Engineering, National Taiwan Ocean University, Keelung City, Chinese Taipei \\ ${ }^{3}$ Department of Computer Science and Information Engineering, Tamkang University, New Taipei City, Chinese Taipei \\ Email: cmown@mail.sju.edu.tw, hawyun.shin@msa.hinet.net, c22424193@gmail.com
}

Received November 20, 2012; revised December 25, 2012; accepted January 8, 2013

\begin{abstract}
The interaction between human and physical devices and devices in the real world is gaining more attention, and requires a natural and intuitive methodology to employ. According to this idea and living well, life has been a growing demand. Thus, how to raise pets in an easy way has been the main issue recently. This study examines the ability of computation, communication, and control technologies to improve human interaction with pets by the technology of the Internet of Things. This work addresses the improvement through the pet application of the ability of location-awareness, and to help the pet owners raise their pet on the activity and eating control easily. Extensive experiment results demonstrate that our proposed system performs significantly help on the kidney disease and reduce the symptoms. Our study not only presents the key improvement of the pet monitor system involved in the ideas of the Internet of Things, but also meets the demands of pet owners, who are out for works without any trouble.
\end{abstract}

Keywords: Internet of Things; Wireless Sensor Network; Pet System

\section{Introduction}

Over the past half century, computers have exponentially increased in processing power and simultaneously decreased in both size and price. This rapid change indicates that computers participate in more daily activities in society. Computers are becoming smaller and cheaper, and the single-purpose computers with embedded sensors are practical from both economical and theoretical viewpoints. Wireless sensor networks have attracted increasing attention and play a vital role in connecting everything in our lives.

Today users are more and more immersed into a complex ubiquitous information. To capture handy information, make use of context information and interact directly with the physical object is the main request in our modern world with the mobile client. That's the main issue of the internet of things (IoT). The IoT provides a set of standards and methodologies to associate object in the real world. There are several approaches for the provision of applications that make such interactions as possible [1,2]. In 2000, the term IoT appears to have been coined by a member of the RFID developer, who referred as the discovering tag information corresponds to a particular RFID. Since that time, people have sized on the phrase "Internet of Things" to refer as the general connection of things, especially to those objects, that are readable, recognizable, locatable, addressable, and controllable via the Internet [3]. In [4], Kranz et al. developed various prototypes to explore novel ways for human computer interaction to realize the appliance of IoT. For example, they make some prototypes to demo the context-aware kitchen utilities, included an instrumented cutting board, vision and sound-based activity detection system, activity recognition using body-worn sensors, and a sensor-augmented knife net gets to infer the context and activities occurring in the kitchen. In addition, the intelligent environment can learn, recognize, and use behavior to provide a variety of novel services to household members. Besides, the authors used policy-aware object design to develop health and safety-aware smart objects as an appliance of IoT, which acts as a smart barrel with embedded storage rules for various chemicals. Depending on temperature, vibration, and barrels' relative proximity, it informs workers about safety violations and prompts them to take appropriate action [5]. Hence, by embedding computational capability's sin all kinds of objects and living objects, IoT will be possible to provide a qualitative and quantitative leap in several sectors: healthcare, logistics, education, entertainment, and so on. In fact, one of the most import elements in the IoT is wireless sensor networks (WSNs) and the extension of ubiquitous computing [6].

WSNs consist of a large number of unattended, selforganized microsensors, which are scattered in an area for a specific application. Each microsensor senses data from the environment, performs simple computations, 
and transmits the data over a wireless medium either directly to the command center or through a cluster gateway. Although WSNs are similar to networks, they differ from networks for their energy constraints, markedly larger density of sensor nodes, lower cost, and precise design for information-gathering [7]. The current trend is to move away from proprietary standard to embrace IPbased sensor networks using the 6LoWPAN/IPv6. This allows the peer connectivity between WSNs and Internet, which is enabling smart objects to participate to the IoT. This integrated factor plays a significant role in our study.

Because of the low birth rate in Taiwan, researchers should focus more on the rapid changes in lifestyle. Some studies indicate that when people go to a park on the weekend, more of them are walking dogs than carrying babies. According to a June 2006 report from Pet Care Services in the United States, America's pet care service generated approximately 363 million USD in revenue in 2005. The annual growth rate of this service should reach $6 \%$ in the future [8]. In addition, Eastern Europe and Asia will become potential markets for pet services. Meadows and Flint indicated that a low birth rate and weakening links between family members have increased the importance of pets [9], leading to a corresponding rise in pet services.

This study attempts to improve pet appliances with the ability of location-awareness, and to help pet owners raise their pets easily. Although numerous automatic pet monitoring systems exist, such as automatic pet doors and pet feeders, these systems cannot meet the needs of pet owners. For example, most pet care systems are based on infrared detector/recognition, which can be used to spot pets at the door, register their movements, and alert owners when pets enter areas where they are not allowed. This infrared detector design has some disadvantages because infrared detectors can be influenced easily by various factors. The detection cannot be correctly recognized. According to the author's previous study, own has built a prototype of the pet system with the cyber-physical-system idea [10]. Herein, the author implements the system with the IoT infrastructure and applied the case study on cat raising.

Accordingly, the organization of this paper is as follows. A brief review of pet products and WSN platforms are given in Section 2. The proposed system modeling is presented in Section 3 while the experiment and analysisare discussed in Section 4. Finally, the conclusion is presented in Section 5.

\section{Preliminaries}

\subsection{Pet Care Solution}

As Taiwanese society continues to have a low birth rate and an aging population, increasingly more people regard their pets as family members. This trend is reflected in pet-related products and activities. For example, some pet owners have started bringing their pets with them while traveling. A report from the 2010 Asia Pacific Pet Economic Conference mentioned that the pet industry has grown considerably in recent years [11]. They forecasted that the market would double in the following two years. Families in Taiwan raised 1,630,000 dogs than in 1999. The family average has $1.55 \%$ dogs in 2007 . Conversely, only 195,000 cats were in families in 2001, and the total increased to 281,000 in 2006 [9]. This is an increase of $4.4 \%$ per family, and we concluded the average family has $1.6 \%$ cats. According to a report of the council for economic planning and development, more than 166,000 babies were born in Taiwan in 2009, representing a drop of more than 20,000 from the previous year [9]. Thus, Taiwan's birthrate has dropped to the world's lowest at $8.29 \%$, with only under one baby born per woman over a lifetime. The average family has more pets than children. This means that the demand for pet products will grow quickly, and household spending on pets will exceed that for children. The pet industry and pet owners have gradually begun to realize the demand for automated raising devices.

Pet doors are one of the most common products in the market, and various types of pet doors are available for cats and dogs. Pet doors can be fitted in a lower portion of a wall or an existing full-sized door. A pet door may consist simply of a flap hung from a horizontal axis. This flap swings open, against the force of gravity, when pushed by an animal. A simple latch may hold the door in a closed position to prevent the movement of the door in either direction. The problem with this simple construction is that any animal small enough to fit through the opening may gain entry or egress, depending on the position of the latch. To prevent passage of unwanted stray animals, electronic pet doors have been designed with magnetically operable latches. In this type of design, any magnetic tag of adequate field strength can unlock the latch.

Pets face many of the same problems as humans, such as obesity, diabetes, and stomach problems. Automated feeding machines can provide for the care of such pets. Several automated pet feeders on the market are capable of dispensing kibbles, and some are capable of feeding canned food. Feeding kibbles prevent food spoilage, whereas feeding canned food does. Canned food cannot be left in a device for prolonged periods because it spoils. Besides, pets also tend to be restless when owners are not at home, and they become hyperactive when owners return. It is occasionally necessary for pet owners to reinforce pet behavior by providing food for certain behaviors they may want the pet to perform. Automated feed- 
ing machines is just invented for pet owners demand.

There are many automatic pet feeders for feeding pets at predetermined times during an owner's absence. This type of pet feeder comprises a base, a feeding bowl with pie-shaped divisions, a timer module, a bowl cover, and a locking mechanism to hold the entire unit in place. The timer provides programmed feeding schedules that determine the time the bowl cover closes or opens. This programming can be achieved through the timer interface or RF and IR remote controllers.

\subsection{The Technology and Architecture of IoT}

The "things" in the IoT not only be referred as "connected together", but also can be realized the functions of recognition, localization, tracing, management and so on. IoT requires all things must can be identified. Generally, the RFID technology is the "speaking technology" for these things on IoT. The RFID technology possessed an outstanding status in the IoT technology. A typical RFID system consists of RFID tag, reader and the application. An agreement from code data preserved in the RFID tag, it can mark objects uniquely. However, without the battery support, the serious disadvantage is the short communication distance of the passive HF RFID tag. Thus, in our study, for the purpose to extend the communication distance, we used the sensor network tags for instead.

To describe the IoT with a technical perspective, the relative study derived the IoT with three parts in the 2011 [12]. The first part is the hardware device layer or the sensing layer. This physical layer occupies less space, making it easier to connect practically anything, anywhere, anytime. The technology of WSN is an outstanding advantage of the IoT in this layer, not only can gain objects' environment information, but can also distinguish each object precisely.

The Second part is the infrastructure or the network layer. This layer consists of converged network formed by all kinds of communication network and internet. It has been widely accepted that this part is the maturest part. Besides, the IoT management center and information center are the parts of network layer. That is, the network layer not only has the ability of network operation, but also should improve the ability of information operation. Besides, with millions of devices connected to the Internet, 3G or LTE technology won't be able to work with. Although the $3 \mathrm{G}$ business is growing, the capacity of the mobile networks is acting as a bottleneck. Hence, to take advantage of these opportunities, the WSNs must be open to the wider public.

The third and last part consists of the applications and services that use the vast quantity of information created by the IoT, it's the application layer. The application layer combined with industry expertise to achieve a broad set of intelligent application solutions. This appli- cations also involve creating new business models and very interesting business initiatives in terms of innovation. The main purpose of the IoT application layer is to provide the information services, and it mainly consisted of three parts, IoT client side, data storage module and data inquiry module.

In our study, we follow the IoT architecture to construct our pet monitor system. The hardware of the first part is based on the WSNs. These sensor nodes are varying in size and depend on the size because different sizes of sensor nodes work efficiently in different fields. In the present era, there are many technologies which use for monitoring are completely based on the wireless sensor networking. Some of the important applications are environmental monitoring, traffic control application, weather checking, and regularity checking of temperature [13]. Wireless sensor networks can also use for detecting the presence of vehicles such as motor cycles up to trains. In our system, the pet wears the collar with sensor tag as an identification. Figure 1 is the example of collar design. Our proposed system can organize as the first pet monitoring system based on the WSN.

Besides, the system infrastructure of the second part is designed to meet the needs of the pet owner. General speaking, home is small and pet sets are messy, WSN is the best choice to build the network structure. Thus, the system is open to the wider public; the pet owner can connect to the gateway at home to control/schedule the pet activity. Furthermore, in the final part, our system supports the following services, the first one is the smart pet door, the pet owner can restrict the pet activity from the outdoor to indoor. The second service is the smart pet feeder, with this system help, the pet owner can schedule the open time of the eating bowl remotely. Thus, the pet can eat food on the health and efficiency control.

\section{The Proposed Pet System}

In our study, the first device of the pet monitor system is the smart pet door, which can help the pet owner to con-

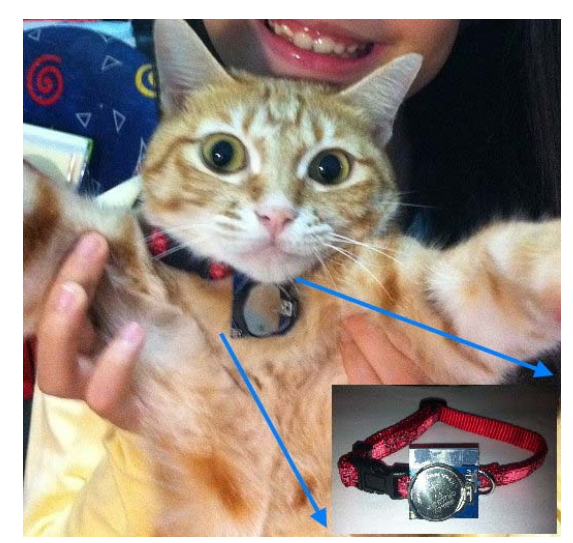

Figure 1. The sensing tag on the collar. 
trol the pet activity. The other device is the smart pet feeder. With the system help, the pet owner can schedule the pet eating bowl time remotely, and make the eating environment on the health and efficiency control.

\subsection{The Smart Pet Door}

Many families install a pet door for their pets. Pet doors are adapted to be fitted in the lower portion of a wall or existing full-sized door. Pet doors allow family pets to pass the door easily. Figure 2(a) shows the system diagram of the pet door. The pet door system consists of one control server, one smart pet door, several environment nodes, and the tag on collars. The pet wears a sensing tag on its collar, and the collar broadcasts the pet ID for every 20 seconds continuously. Figure 1 shows the collar design. The pet door detects the pet's location and periodically requests the pet activity list from the server. Accordingly, when the device on the pet door receives the broadcast packet from the pet tag, the device will measure the estimated distance by the RSSI (Received Signal Strength Indicator) value between them, the measuring equation is listed as follows,

$$
R S S I=-\left(10 n \log _{10} d+A\right)
$$

In (1), $n$ is signal propagation constant or exponent, $d$ is the distance from sender and $A$ is the received signal strength at 1 meter distance [14].

In this study, the proposed system is based on the fol-

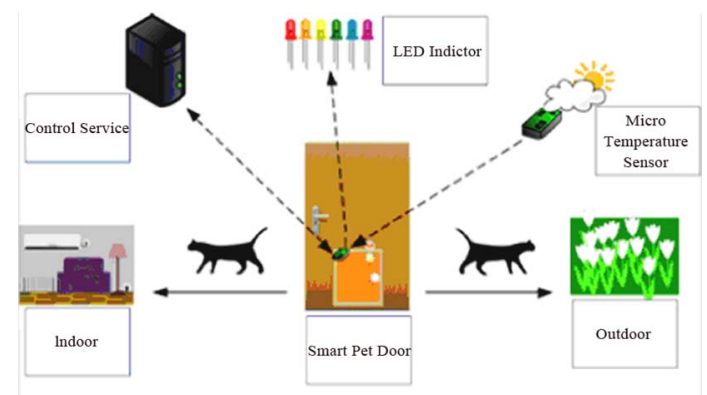

(a)

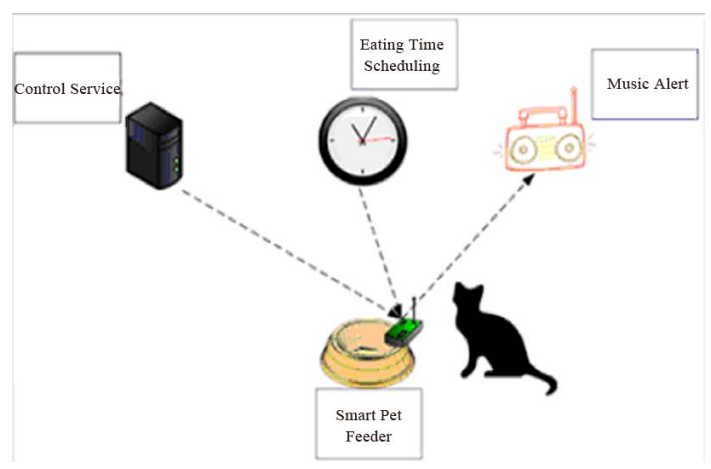

(b)

Figure 2. The diagram of pet system with (a) the smart pet door; (b) the smart pet feeder. lowing rules:

- The pet tag broadcasts the pet ID for every 20 seconds continuously.

- An item identification can detect the animal seeking passage through the pet door. Because the node of WSN is the active tag, the sensing range is about from $1 \mathrm{~m}$ to $2 \mathrm{~m}$. The pet door will unlock when the activity list is permitted.

- The pet owner can set the different passage permission for the different pets. Thus the specific pet, for example, the sick pet, can be forbidden to go outside. The passage permission will store in the control server.

- Aled light can indicate the pet owner where the pet is located. In this case, the pet owner doesn't need to whistle the pet for making sure the location; the led indicator can show the pet location. This indicator costs less power, but can gain more conveniently.

- The environment nodes can report the weather to the server for every 10 minutes, the pet owner can set the weather rules to control the curfew. For example, if it's raining outdoors, the pets must come home but get no permission to go outside.

- If there are more than one pets around the pet door, the pet door will arrange the pet priority by their RSSI values. Then check the activity list from the control server by their sequence.

Figure 3 shows the interior design of the smart pet door. The WSN module is installed on the upper right of the pet door as the control and communication center, which is responsible for receiving the pet id and driving the control motors shown in Figure 3(a). Figures 3(b) and (c) show the tow motors; the tow motors in Figure 3(b) is used to unlock the pet door, and the tow motor in Figure 3(c) controls the swing direction of the flap. Figures 3(d) and (e) show the light motion sensors installed on the top panel over the pet door. These sensors are used to detect the flap swing direction, and the system can use this data to trigger the LED at the top of the pet door, which can indicate the pet location to the pet owner. For example, a red LED indicates that the pet is indoors, whereas a green LED indicates that the pet is outdoors.

We compared the proposed system with the general product of Staywell [15], as shown in Table 1, to identify the differences between these systems. Because the Staywell product is based on magnetic tag sensing, the cost and power supply is lower than those in our smart pet door. However, according to previous discussions, our system is designed based on the support of pet owner demand. The power saving is another advantage of WSN. Hence, our system exhibits dominance.

\subsection{The Smart Pet Feeder}

Most pets eat dry food. Pet owners cannot leave out wet 


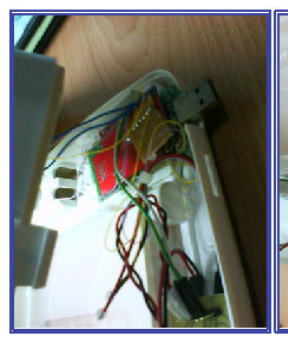

(a)

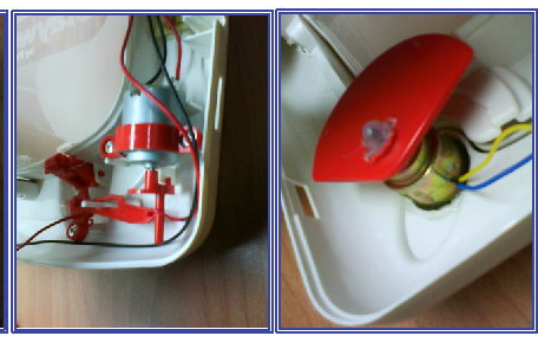

(b) (c)

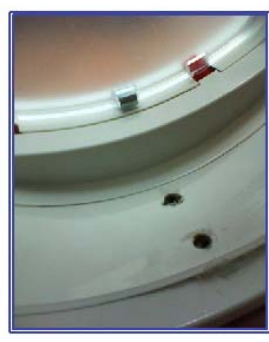

(d)

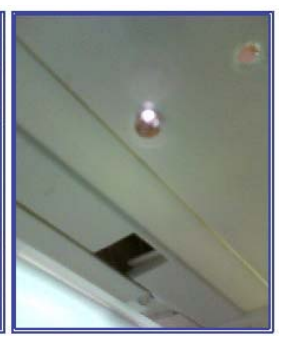

(e)
Figure 3. The outlook of the pet door, (a), (b) and (c) show the tow motors, (d) and (e) are the light motion sensors.

Table 1. The comparison between our proposed smart pet door and the general product.

\begin{tabular}{ccc}
\hline & Smart Pet Door & General Pet Door [15] \\
\hline Cost & Higher & Lower \\
Power Supply & Higher & Lower \\
Pet Tag & Sensor Tag & Magnetic Tag \\
Active Schedule & Support & No \\
Remotely Control & Support & No \\
Position Indicator & Support & No \\
Environment Control & Support & No \\
Pet Identification & Support & No \\
\hline
\end{tabular}

food because it spoils in the time the pet must eat it. Dry food feeders can keep dry food fresh to longer, and allow the owner to feed a pet automatically, even when outside the home. These feeders are nearly always placed on the floor next to an external wall where they are accessible to crawling insects, such as ants, which have a propensity to seek a supply of food. These insects not only contaminate the pet food, but are often intolerable to the pet owner.

Numerous attempts have been made to design a pet feeder that resolves the problem of crawling insects. Most of these efforts have produced feeders with a multiplicity of discrete components. These feeders must be disassembled for cleaning and then reassembled before further use. The pet feeder produced by the trading company has the general appearance of a conventional feeder, but has a moat-forming cavity surrounding the food bowl [16]. This pet feeder can keep the pet food and water clean until the pet is ready to eat. This pet feeder also has a bowl cover that opens and closes automatically. The bowl cover is actuated by an infrared proximity sensor and battery-operated electric motor. The sensor detects the presence of the pet and then opens the cover, enabling only the pet to have access to the food. When the pet is out of sensor range, the bowl cover closes automatically. This keeps dust, flies, and bugs from reaching the food and keeps the food fresh. The pet feeder system consists of on control server, one smart pet feeder, and the tag on collars.

In this study, our proposed smart pet feeder is based on the following design rules:

- The pet tag broadcasts the pet ID for every 20 seconds continuously.

- An item identification can detect the animal seeking passage through the pet feeder. Because the node of WSN is the active tag, the sensing range is about from $1 \mathrm{~m}$ to $2 \mathrm{~m}$. The pet feeder will remove the bowl cover when the eating activity is permitted.

- The pet owner can set the eating permission for one pet to one feeder. Thus, only the granted pet can approach the feeder for food. Besides, most of the pets have deeply locality of animal behavior, share food is not common for them.

- The pet owner can schedule eating time remotely, and prevent the unwelcome insects by closing the bowl cover in the non-eating time.

- The pet owner can record the greeting voice to alert the eating time.

- During the eating time, the pet is free to approach for food.

- If there are more than one pets around the pet feeder, the pet feeder will arrange the pet priority by their RSSI values. Then check the eating activity list from the control server by their sequence.

Figure 4 shows the interior design of the smart pet feeder. Figure 4(a) shows the feeder device. This study referred to the feeder product, which has four bowls. Figure 4(a) also shows the embedded sound playback module, which is used to play the received playback command from the WSN module. Figure 4(b) shows the inside view of the smart pet feeder, in which the WSN module can drive the rotation gear in the middle of the feeder to remove the bowl cover, and is also responsible for receiving the signal of pet id, checking the RSSI value, controlling the voice greeting, and triggering the bowl cover to open or close.

The difference between our proposed smart pet feeder and the product from the Trading Company is shown in Table 2 [14]. As shown in Table 2, our smart pet feeder has a higher cost and larger power supply because of the WSN module. Traditional feeders in the market do not support pet identity recognition, and do not require tags. 


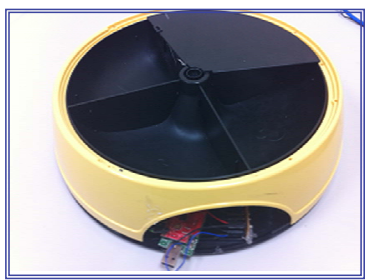

(a)

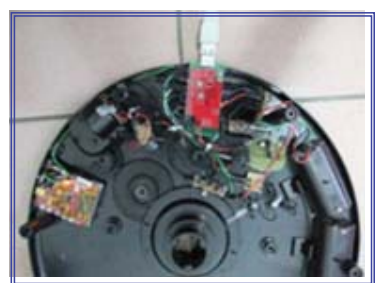

(b)
Figure 4. The outlook of the pet feeder, (a) is the feeder device, (b) is the inside view of the feeder.

Table 2. The comparison between our proposed smart pet feeder and the general product.

\begin{tabular}{ccc}
\hline & Smart Pet Feeder & $\begin{array}{c}\text { General Pet Feeder } \\
{[14]}\end{array}$ \\
\hline Cost & Higher & Lower \\
Power Supply & Need & Need \\
Pet Tag & Need & No \\
Eating Schedule & Full Functional Support & Partly Support \\
Bowl Cover & Full Automatic Support & Partly Support \\
Remotely Control & Support & No \\
Pet Identification & Support & No \\
Voice Greeting & Support & No \\
\hline
\end{tabular}

With the function of identity recognition, our proposed system can fulfill the requirement of pet owners than traditional products. For example, the comparison product supports the setup of an eating schedule in the front panel; however, it lacks the convenience of our system. By contrast, our system enables the owner to set the eating schedule on web page remotely, which can help the pet to moderate its eating habits.

\subsection{The Control Center}

Figures 5(a) and (b) show the proposed management system of the control service. Figure 5(a) is the Window executed program, which can help the pet owner to execute on the PC. A php program can be remotely executed on the mobile device for the pet owner convenience in Figure 5(b). In our management system, the pet owner can upload the pet image to the corresponding pet id. Furthermore, the micro temperature (temperature and humidity), pet activity permission, eating time schedule; all the user defined data can be defined in this software and stored at the control server.

\section{Experiments and Analysis}

Mrs. Lee, who lives in an apartment with a balcony in Taipei, Taiwan, was applied as our experimental case. She has two cats, one of which is young and energetic, and the other is old and has kidney disease. Generally, kidney disease or kidney failure is the most common medical problem experienced by older cats. Several cats with kidney disease can survive for months or years after diagnosis. However, kidney disease is not curable. The disease is chronic and progressive, and results in fatality in most cases.

The goal of treatment for kidney disease is to reduce the symptoms and slow the progression of the disease. The main forms of treatment are dietary change and fluid therapy, and dry food is not recommended. A low-protein diet was previously recommended to help to reduce the workload of the ailing kidneys. Thus, in the past, the old cat was isolated in a cage because of the distinct treatments for both cats. However, Mrs. Lee desired superior treatment for her cats. Therefore, we installed our system in her apartment as a one-month experiment.

Mrs. Lee previously installed a small pet door for her cats. We installed our smart pet door on the sliding door to the balcony. Our smart pet door enabled the cats to go to outdoors, and the activity schedule restricted the older

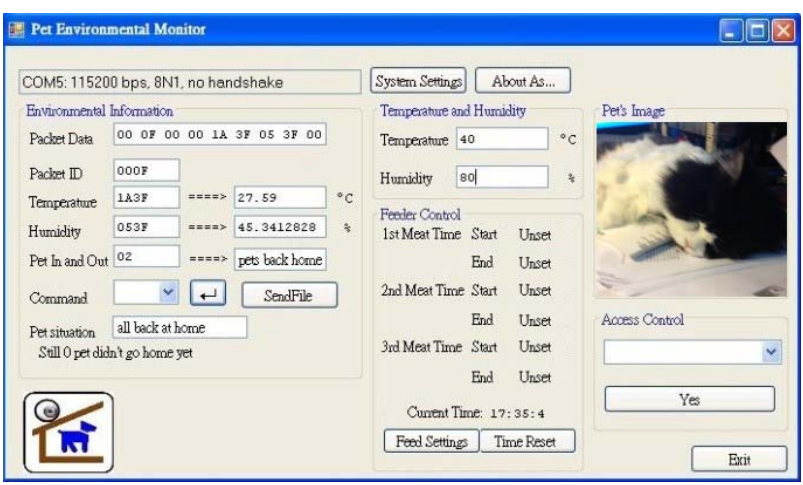

(a)

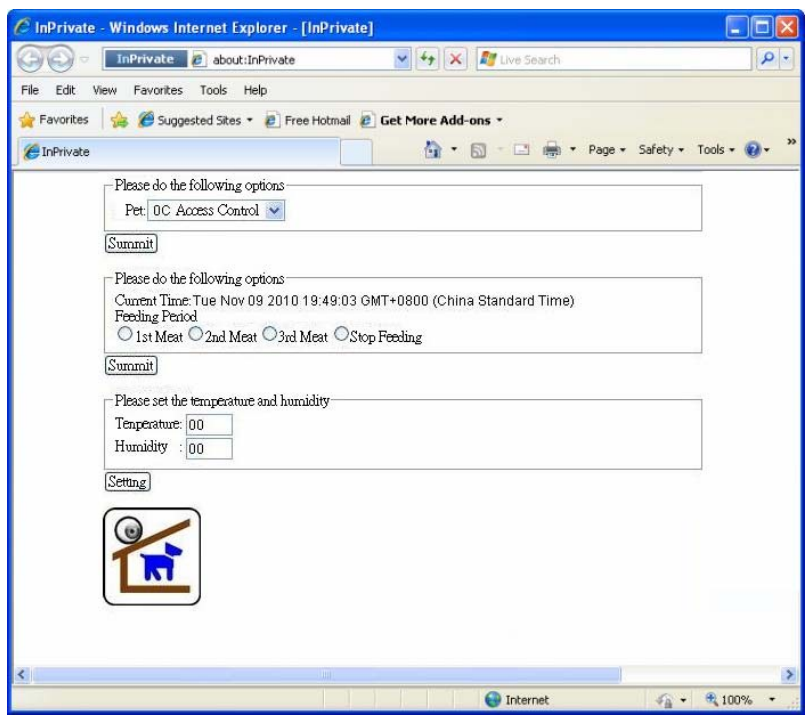

(b)

Figure 5. (a) The System management for windows; (b) The system management program for web. 


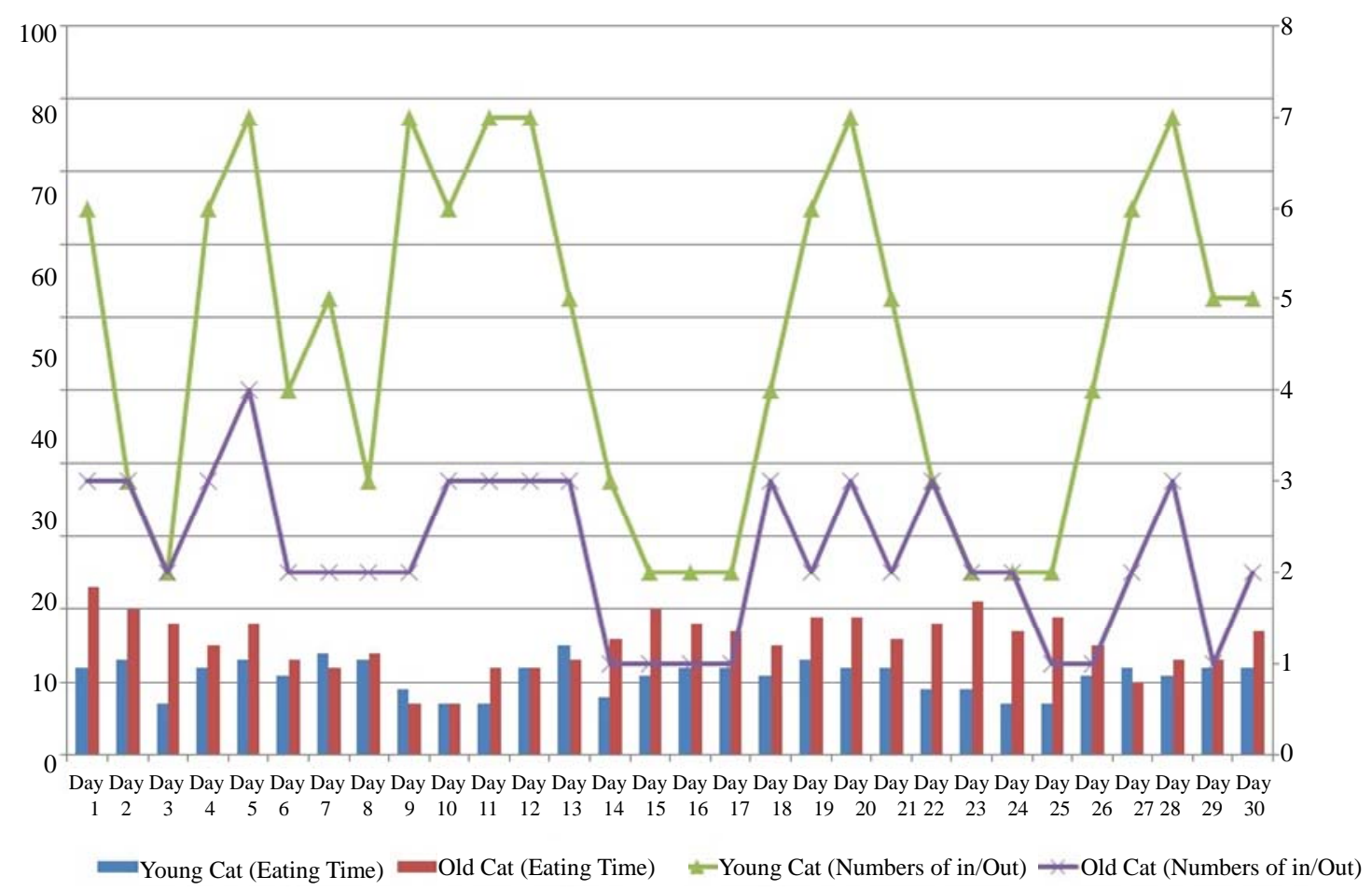

Figure 6. The experiment of two cats in the Mrs. Lee apartment.

cat from remaining on the balcony for long periods. Our system provided two smart pet feeders for both cats, which supplied them with different meals at each feeding time. We record the eating time for each cat as the illness tracking. The cats wore different pet tags for identity recognition.

Figure 6 shows the experimental results of the two cats in Mrs. Lee's apartment. With the help of the smart pet care system, we collected in and out numbers of the apartment and the eating times of the two meals for both cats. Because the old cat had kidney disease and was restricted to the balcony at night, the number of ins/outs was lower than those of the young cat (The average ins and outs were 4.6 per day for the young cat, and the average number was 2.2 for the old cat). The prescription food for the old cat was not as appealing as the normal food; therefore, the eating time was longer (The average eating time was 10.8 minutes per day for the young cat, and the eating time of the old cat was 15.5 minutes per day).

\section{Conclusions and Future Work}

The interaction between humans and physical devices and objects is attracting increasing attention. Many studies have attempted to provide a natural and intuitive approach to request services. The current trend of combining pet control and IoT technology offers exciting future developments. The proposed system is also referred on smart-home technology, including the smart pet door and pet feeder. The results not only present the key improvement of the pet monitor system involved in the IoT technology, but also meet the demand of pet owners.

The basic vision behind the IoT, it may have a new way of operational method, it may have a new method of connecting devices, and there might be the even complete clean-slate approach. As the full operational definition is not yet finalized, there are numerous research issues that can be worked on. As a next step, we will fully integrate the other pet care devices into our system, including litter boxes, pet cam, etc. With that, the diverse needs of the owners can be met, and the health, monitor, and entertainment topics for pets are all covered. Besides, standing as the cloud term, how to connect the numerous networking devices around the globe is the next issue. In the future, we will centralize on the study of the IoT gateway and long distance detection of the pets.

\section{Acknowledgements}

The author thanks the National Science Council of the Republic of China, Taiwan for partially supporting this research under Contract No. NSC 101-2221-E-129-014.

\section{REFERENCES}

[1] M. Rohs and B. Gfeller, "Using Camera-Equipped Mobile Phones for Interacting with Real-World Object," Proceedings of Advances in Pervasive Computing, April 2004, pp. 265-271. 
http://catalog.hathitrust.org/Record/004761231

[2] C. Sammarco and A. Lera, "Improving Service Management in the Internet of Things," Sensors, Vol. 12, No. 9, 2012, pp. 11888-11909. doi:10.3390/s120911888

[3] H. Ning and H. Liu, "Cyber-Physicl-Social Based Security Architecture for Future Internet of Things,” Advanced in Internet of Things, Vol. 2, No. 1, 2012, pp. 1-7. doi:10.4236/ait.2012.21001

[4] M. Kranz, P. Holleis and A. Schmidt, "Embedded Interaction Interacting with the Internet of Things," IEEE Internet Computing, Vol. 14, No. 2, 2010, pp. 46-53. doi:10.1109/MIC.2009.141

[5] G. Kortuen, F. Kawsar, D. Fitton and V. Sundramoorthy, "Smart Objects as Building Blocks for the Internet of Things,” IEEE Internet Computing, Vol. 14, No. 1, 2010, pp. 44-51. doi:10.1109/MIC.2009.143

[6] I. F. Akyildiz, W. Su, Y. Sankarasubramaniam and E. Cayirci, "Wireless Sensor Networks: A Survey," Computer Networks, Vol. 38, No. 4, 2002, pp. 393-422. doi:10.1016/S1389-1286(01)00302-4

[7] A. L. Bleda, A. J. Jara, R. Maestre, G. Santa and A. F. G. Skarmeta, "Evaluation of the Impact of Furniture on Communications Performance for Ubiquitous Deployment of Wireless Sensor Networks in Smart Homes,"
Sensors, Vol. 12, No. 5, 2012, pp. 6463-6496. doi:10.3390/s120506463

[8] APPEC, Asia Pacific Pet Economic Conference. http://www.2010appec.org

[9] Packaged Facts, "Pet Care Services in the US," 2nd Edition, 2006.

[10] C.-M. Own, "For the Pet Care Appliance of Location Aware Infrastructure on Cyber Physical System,” International Journal of Distributed Sensor Networks, Vol. 2012, 2012, 8 Pages.

[11] Council for Economic Planning and Development 2009. http://www.cepd. gov.tw/

[12] G. Feller, “The Internet of Things: In a Connected World of Smart Objects,” Accenture \& Bankinter Foundation of Innovation, 2011.

[13] J. Yick, B. Mukherjee and D. Ghosal, "Wireless Sensor Network Survey,” Computer Networks, Vol. 52, No. 12, 2008, pp. 2292-2330. doi:10.1016/j.comnet.2008.04.002

[14] Chipcon: CC2420 802.15.4 Compliant Radio. http://www.chipcon.com.

[15] The Staywell International. http://www.staywell.co.uk

[16] Automatic Pet Feeder. http://gadgets.softpedia.com/ 